\title{
Perturbed Lepton-Specific Two-Higgs-Doublet Model Facing Experimental Hints for Physics beyond the Standard Model
}

\author{
Andreas Crivellin \\ CERN Theory Division, CH-1211 Geneva 23, Switzerland \\ Julian Heeck \\ Service de Physique Théorique, Université Libre de Bruxelles, Boulevard du Triomphe, CP225, 1050 Brussels, Belgium \\ Peter Stoffer \\ Helmholtz-Institut für Strahlen- und Kernphysik (Theory) and Bethe Center for Theoretical Physics, \\ University of Bonn, D-53115 Bonn, Germany
}

(Received 10 August 2015; published 23 February 2016)

\begin{abstract}
$B A B A R$, Belle, and $\mathrm{LHCb}$ Collaborations report evidence for new physics in $B \rightarrow D \tau \nu$ and $B \rightarrow D^{*} \tau \nu$ of approximately $3.8 \sigma$. There is also the long lasting discrepancy of about $3 \sigma$ in the anomalous magnetic moment of the muon, and the branching ratio for $\tau \rightarrow \mu \nu \nu$ is $1.8 \sigma(2.4 \sigma)$ above the standard model expectation using the HFAG (PDG) values. Furthermore, CMS Collaboration finds hints for a nonzero decay rate of $h \rightarrow \mu \tau$. Interestingly, all these observations can be explained by introducing new scalars. In this Letter we consider these processes within a lepton-specific two-Higgs doublet model (i.e., of type X) with additional nonstandard Yukawa couplings. It is found that one can accommodate $\tau \rightarrow \mu \nu \nu$ with modified Higgs $-\tau$ couplings. The anomalous magnetic moment of the muon can be explained if the additional neutral $C P$-even Higgs boson $H$ is light (below $100 \mathrm{GeV}$ ). Also $\mathcal{R}(D)$ and $\mathcal{R}\left(D^{*}\right)$ can be easily explained by additional $t-c-$ Higgs couplings. Combining these $t-c$ couplings with a light $H$ the decay rate for $t \rightarrow H c$ can be in a testable range for the LHC. Effects in $h \rightarrow \mu \tau$ are also possible, but in this case a simultaneous explanation of the anomalous magnetic moment of the muon is difficult due to the unavoidable $\tau \rightarrow \mu \gamma$ decay.
\end{abstract}

DOI: 10.1103/PhysRevLett.116.081801

Introduction.-In addition to direct searches for new physics (NP) beyond the standard model (SM) performed at high energies at the LHC, low-energy precision flavor observables provide a complementary window to NP. The anomalous magnetic moment of the muon $(g-2)_{\mu}$ is a prime example as it is very sensitive to physics beyond the SM entering via quantum corrections. Also, tauonic $B$ meson decays and $\tau \rightarrow \mu \nu \nu$ are excellent probes of NP: they test lepton flavor universality and are sensitive to new degrees of freedom that couple proportional to the mass of the involved particles (e.g., Higgs bosons [1]) because of the heavy $\tau$ lepton involved. The observation of flavorviolating decays of the SM Higgs boson, e.g., $h \rightarrow \mu \tau$, would prove the existence of NP.

Let us briefly review the current experimental and theoretical situation in these processes. The world average of the measurement of $a_{\mu} \equiv(g-2)_{\mu} / 2$ is completely dominated by the Brookhaven experiment E821 [2] and is given by [3]

Published by the American Physical Society under the terms of the Creative Commons Attribution 3.0 License. Further distribution of this work must maintain attribution to the author(s) and the published article's title, journal citation, and DOI.

$$
a_{\mu}^{\exp }=(116592091 \pm 54 \pm 33) \times 10^{-11},
$$

where the first error is statistical and the second is systematic. The current SM prediction is [4-12]

$$
a_{\mu}^{\mathrm{SM}}=(116591855 \pm 59) \times 10^{-11},
$$

where almost the whole uncertainty is due to hadronic effects. This amounts to a discrepancy between the SM and the experimental value of $a_{\mu}^{\exp }-a_{\mu}^{\mathrm{SM}}=(236 \pm 87) \times$ $10^{-11}$, i.e., a $2.7 \sigma$ deviation. It is not yet clear if this discrepancy is due to NP or rather underestimated hadronic uncertainties; there are ongoing efforts to reduce the model dependence in the hadronic light-by-light estimate based on dispersion relations [13-16] or lattice QCD [17-20]. Possible NP explanations besides supersymmetry (see for example Ref. [21] for a review) include very light $Z^{\prime}$ bosons [22-29], leptoquarks [30,31], and additional fermions [32] but also new scalar contributions in twoHiggs-doublet models (2HDMs) [33,34], also within the lepton-specific 2HDM [35-37].

Concerning tauonic $B$ decays, $B A B A R$ Collaboration performed an analysis of the semileptonic $B$ decays $B \rightarrow$ $D \tau \nu$ and $B \rightarrow D^{*} \tau \nu$ using the full available data set [38]. 
Recently, these decays have also been reanalyzed by Belle Collaboration [39,40], and LHCb Collaboration measured the mode $B \rightarrow D^{*} \tau \nu$ [41]. These experiments determine the ratios

$$
\mathcal{R}\left(D^{(*)}\right) \equiv \mathrm{BR}\left(B \rightarrow D^{(*)} \tau \nu\right) / \mathrm{BR}\left(B \rightarrow D^{(*)} \ell \nu\right),
$$

where $\ell=e$ or $\mu$. Combining the data, one finds [42]

$$
\mathcal{R}(D)_{\exp }=0.388 \pm 0.047, \quad \mathcal{R}\left(D^{*}\right)_{\exp }=0.321 \pm 0.021 .
$$

Comparing these values to the SM prediction $[38,43,44]$

$$
\mathcal{R}(D)_{\mathrm{SM}}=0.297 \pm 0.017, \quad \mathcal{R}\left(D^{*}\right)_{\mathrm{SM}}=0.252 \pm 0.003,
$$

we see that there is a discrepancy of $1.8 \sigma$ for $\mathcal{R}(D)$ and $3.3 \sigma$ for $\mathcal{R}\left(D^{*}\right)$; combining them gives a $3.8 \sigma$ deviation from the SM (compared to $3.4 \sigma$ taking into account the $B A B A R$ results only [38]). Models solving the $\mathcal{R}(D)$ puzzle have been discussed extensively in the literature [45-59], including the possibility of charged scalars $[46,48,49,60]$.

For $\tau \rightarrow \mu \nu \nu$, the dominant uncertainty in the SM prediction for the branching ratio comes from the $\tau$ lifetime $\tau_{\tau}$. Using the PDG [3] values for the $\tau$ lifetime, $\tau_{\tau}=(290.3 \pm 0.5) \times 10^{-15} \mathrm{~s}$, and branching ratios

$$
\begin{aligned}
& B_{\mu} \equiv \operatorname{BR}(\tau \rightarrow \mu \bar{\nu} \nu)_{\exp }=(17.41 \pm 0.04) \%, \\
& B_{e} \equiv \operatorname{BR}(\tau \rightarrow e \bar{\nu} \nu)_{\exp }=(17.83 \pm 0.04) \%,
\end{aligned}
$$

we can determine the deviations $\Delta_{\ell} \equiv B_{\ell} / B_{\ell}^{\mathrm{SM}}-1$ [61] from the SM prediction to be

$$
\Delta_{\mu}^{\mathrm{PDG}}=(0.69 \pm 0.29) \%, \quad \Delta_{e}=(0.28 \pm 0.28) \% .
$$

There is a deviation of about $2.4 \sigma$ in the muon data, whereas the electron channel is compatible with the SM prediction. HFAG finds essentially the same value for the $\tau$ lifetime, but a slightly lower $\operatorname{BR}(\tau \rightarrow \mu \bar{\nu} \nu)_{\exp }=(17.39 \pm$ $0.04) \%$ [62], alleviating the deviation to approximately $2 \sigma$ :

$$
\Delta_{\mu}^{\mathrm{HFAG}}=(0.59 \pm 0.32) \% .
$$

Again, charged Higgs bosons $[61,63,64]$ but also neutral $Z^{\prime}$ bosons [65] affect this decay.

Moving from lepton nonuniversality to outright lepton flavor violation, we are drawn to the recent CMS excess of $2.4 \sigma$ in $h \rightarrow \mu \tau$ [66]:

$$
\mathrm{BR}(h \rightarrow \mu \tau)=\left(0.84_{-0.37}^{+0.39}\right) \% .
$$

Possible explanations naturally require an extended Higgs sector [34,67-73].

As we see from the previous discussion, all of the discrepancies outlined above can be solved by additional scalars. A $2 \mathrm{HDM}$ is a good candidate since it introduces a charged scalar $H^{+}$and two neutral states $H$ and $A$ beyond the SM. 2HDMs have been studied for many years with a focus on the type-II models [74-76]. However, there are also other models without flavor-changing neutral currents at the tree level, i.e., type I, type X (lepton specific) and the flipped 2HDM (see Ref. [77] for a review). More general models with flavor-changing neutral Higgs couplings at the tree level are named type-III models. Here, the focus has been on minimal flavor violation [78-80], alignment $[81,82]$, or natural flavor conservation $[79,83]$, but generic 2HDMs of type III have also been studied [49]. In these models type II is recovered in the absence of flavorchanging neutral Higgs couplings. While the type-II model cannot explain $\mathcal{R}(D)$ and $\mathcal{R}\left(D^{*}\right)$ simultaneously [38], this can be achieved by supplementing the model with additional nonholomorphic couplings [46]. However, this model (as the normal type-II model) is under pressure from $b \rightarrow s \gamma$ data [84] and LHC searches for $A \rightarrow \tau \tau$ [85]. Furthermore, no sizable effect in the anomalous magnetic moment of the muon can be generated [49], and also explaining $h \rightarrow \mu \tau$ is challenging.

Therefore, we choose to consider here the 2HDM of type $\mathrm{X}$ (lepton specific), where the heavy Higgs couplings to quarks are suppressed by $\tan \beta$ compared to the type-II model and the bounds from $b \rightarrow s \gamma$ and LHC searches are much weaker, leaving more space for effects in $(g-2)_{\mu}$ and tauonic $B$ decays. Furthermore, the sign of the coupling of heavy Higgs bosons to the $\tau$ lepton can be reversed, allowing for constructive interference in $\tau \rightarrow \mu \nu \nu$. Large effects in $h \rightarrow \mu \tau$ are possible, compared to the type-II-like model, as the Barr-Zee contributions to $\tau \rightarrow \mu \gamma$ involving quarks are suppressed.

2HDM-X.-We will study a lepton-specific 2HDM $(2 \mathrm{HDM}-X)$, defined by the Yukawa couplings in the Lagrangian

$$
\mathcal{L}_{Y}=-\bar{Q}_{L} Y^{u} \tilde{\Phi}_{2} u_{R}-\bar{Q}_{L} Y^{d} \Phi_{2} d_{R}-\bar{L}_{L} Y^{\ell} \Phi_{1} e_{R}+\text { H.c. }
$$

with additional couplings that break the type- $\mathrm{X}$ structure

$$
\Delta \mathcal{L}_{Y}=-\bar{Q}_{L} \xi^{u} \tilde{\Phi}_{1} u_{R}-\bar{Q}_{L} \xi^{d} \Phi_{1} d_{R}-\bar{L}_{L} \xi^{\ell} \Phi_{2} e_{R}+\text { H.c. }
$$

For arbitrary matrices $Y^{u, d, \ell}$ and $\xi^{u, d, \ell}$ this parametrizes the (type-III) 2HDM with the general Yukawa interactions. We will however assume the coupling structure to be close to type $\mathrm{X}$, i.e., the $\xi$ matrices to be perturbations. After electroweak symmetry breaking the following field redefinitions are necessary in order to render the fermion mass matrices diagonal

$$
d_{P} \rightarrow D_{P}^{\dagger} d_{P}, \quad u_{P} \rightarrow U_{P}^{\dagger} u_{P}, \quad \ell_{P} \rightarrow L_{P}^{\dagger} \ell_{P},
$$

where $P=L, R$. We define the (nondiagonal) matrices

$$
\epsilon^{u} \equiv U_{L}^{\dagger} \xi^{u} U_{R}, \quad \epsilon^{d} \equiv D_{L}^{\dagger} \xi^{d} D_{R}, \quad \epsilon^{\ell} \equiv L_{L}^{\dagger} \xi^{\ell} L_{R},
$$




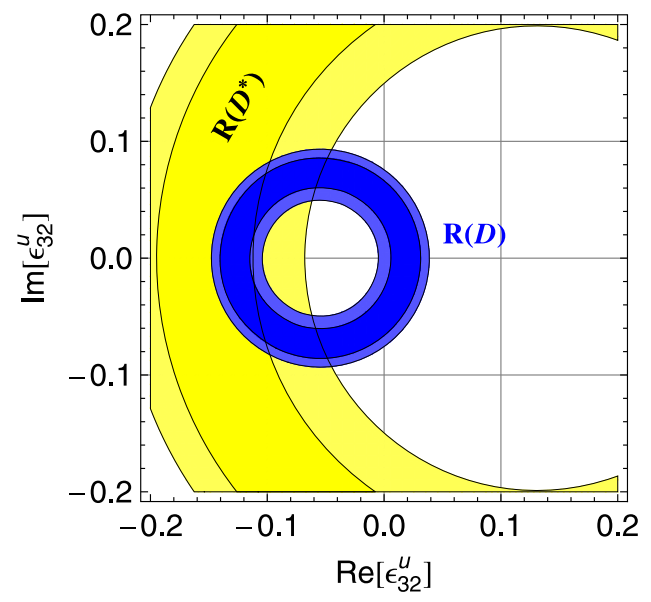

FIG. 1. Allowed regions in the $\epsilon_{32}^{u}$ plane from $\mathcal{R}(D)$ (blue) and $\mathcal{R}\left(D^{*}\right)$ (yellow) for $\tan \beta=50, m_{H^{+}}=200 \mathrm{GeV}$, and $\epsilon_{33}^{\ell}=0$. $\epsilon^{u}$ is given at the matching scale $m_{H^{+}}$.

and express the Yukawa couplings in terms of the physical masses and the couplings $\epsilon$ (the 2HDM- $X$ model is recovered in the limit $\epsilon \rightarrow 0$ ).

We are not concerned with the issue of neutrino masses in this Letter, and hence do not introduce, e.g., right-handed neutrinos. The explicit expressions for the Higgs interactions with fermions are given in the Supplemental Material [86]. Note that for $\epsilon_{33}^{\ell}>m_{\tau} / v$, where $v \simeq$ $174 \mathrm{GeV}$ is the vacuum expectation value, the sign of the couplings of $A, H$, and $H^{+}$to taus is reversed. This will be important later as in this way the sign of the contribution to $\tau \rightarrow \mu \nu \nu$ can be flipped. In our notation, $h$ is the SM-like Higgs boson, and $H$ and $A$ are the additional $C P$-even and $C P$-odd Higgs bosons; due to the mainly leptophilic couplings of $H$ and $A$, collider bounds on their masses are quite weak and they can be even lighter than the SMlike scalar boson. In particular, $\sin (\beta-\alpha)=1$ always corresponds to the SM-like limit in our notation, even for $m_{H}<m_{h}$.

In the following, we will assume $\epsilon^{d}=0$ and take $\epsilon^{u}$ and $\epsilon^{\ell}$ to be of the form

$$
\epsilon^{u}=\left(\begin{array}{ccc}
0 & 0 & 0 \\
0 & 0 & 0 \\
0 & \times & \times
\end{array}\right), \quad \epsilon^{\ell}=\left(\begin{array}{ccc}
0 & 0 & 0 \\
0 & 0 & 0 \\
0 & \times & \times
\end{array}\right),
$$

where $\times$ denotes a (sizable) nonzero entry. Our structure for $\epsilon^{u}$ and $\epsilon^{d}$ is motivated by the hierarchy of the Yukawa couplings and the CKM matrix in the SM. Since the top coupling is large and left-handed rotations are known to be small, we assume only $\epsilon_{33}^{u}$ and $\epsilon_{32}^{u}$ to be sizable. We do not consider effects in $\epsilon^{d}$ as also the down-type Yukawa couplings are small. This agrees very well with phenomenology: off-diagonal elements in $\epsilon^{d}$ are bounded by flavor-changing neutral currents [49] and $\epsilon_{13}^{u}\left(\epsilon_{23}^{u}\right)$ is severely constrained from $b \rightarrow d(s) \gamma$ [105]. In the lepton sector, this structure avoids lepton-flavor violation involving electrons (i.e., bounds from $\mu \rightarrow e \gamma, \mu \rightarrow e e e$, and $\mu \rightarrow e$ conversion in nuclei), but still allows for effects in $a_{\mu}, \tau \rightarrow \mu \nu \nu$, and even $h \rightarrow \mu \tau$.

Phenomenological analysis.-Using the formulas collected in Ref. [86] as supplemental material, we study the phenomenology of our 2HDM and show that it can indeed resolve the anomalies outlined in the Introduction.

$\mathcal{R}(D)$ and $\mathcal{R}\left(D^{*}\right)$ : Let us first consider $\mathcal{R}(D)$ and $\mathcal{R}\left(D^{*}\right)$. From Fig. 1 we see that $\mathcal{R}(D)$ and $\mathcal{R}\left(D^{*}\right)$ can be explained simultaneously for negative values of $\epsilon_{32}^{u}$ with a small or vanishing imaginary part. Note that sizable values of $\epsilon_{32}^{u}$ are required, i.e., of the order of $10^{-1}$. This is important for $t \rightarrow H c$ to be considered later.

$\tau \rightarrow \ell \nu \nu$ : The tree-level charged Higgs contribution interferes destructively with the SM for $\epsilon_{33}^{\ell}=0$. However, for $\epsilon_{33}^{\ell}>m_{\tau} / v$ the interference is constructive, allowing for an explanation of the PDG data, which is in more than a $2 \sigma$ tension with the SM. The one-loop contributions interfere again destructively (independently of $\epsilon_{33}$ ) and are important for nondegenerate $A$ and $H$ masses. Nonetheless, even if $m_{H}=30 \mathrm{GeV}$ and $m_{A}=200 \mathrm{GeV}$, the values $m_{H^{+}}=200 \mathrm{GeV}, \epsilon_{33}^{\ell}=$ $2 m_{\tau} / v$, and $\tan \beta>60$ are consistent with data (see Fig. 2). Furthermore, as one can also see from Fig. 2, for $\epsilon_{32}^{u} \simeq 0.1$ also $\mathcal{R}(D)$ and $\mathcal{R}\left(D^{*}\right)$ can be brought into agreement with the measurements. Therefore, the possibility to flip the sign of the $H, A$ coupling to taus allows us to have smaller values of $\tan \beta$ than in the 2HDM- $X$ model.

Anomalous magnetic moment of the muon: In $(g-2)_{\mu}$ the one-loop and the two-loop Barr-Zee contribution have

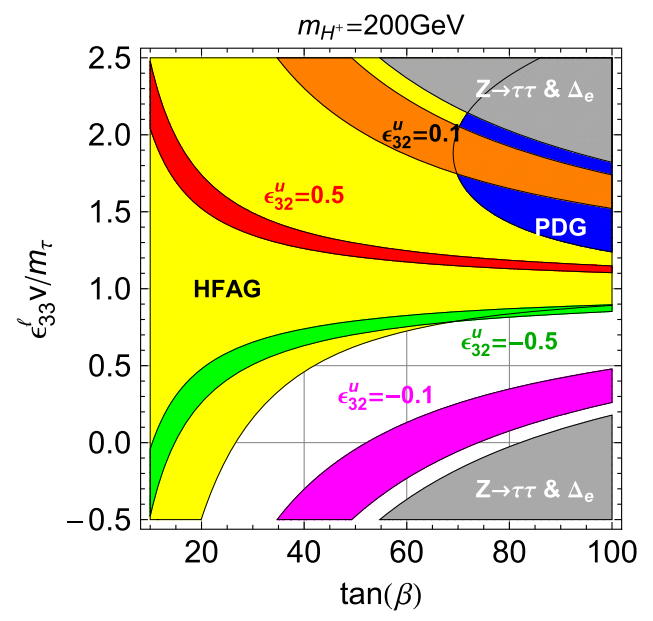

FIG. 2. Allowed regions in the $\tan \beta-v / m_{\tau} \epsilon_{33}^{\ell}$ plane from $\mathcal{R}\left(D^{(*)}\right)$ and $\tau \rightarrow \mu \nu \nu$ at the $2 \sigma$ level. The yellow region is allowed by $\tau \rightarrow \mu \nu \nu$ using the HFAG result for $m_{H}=30 \mathrm{GeV}$ and $m_{A}=200 \mathrm{GeV}$, while the (darker) blue one is the allowed region using the PDG result. The red, orange, green, and magenta bands correspond to the allowed regions by $\mathcal{R}\left(D^{(*)}\right)$ for different values of $\epsilon_{32}^{u}$. The gray region is excluded by $Z \rightarrow \tau \tau$ and $\tau \rightarrow e \nu \nu$. For $m_{H} \simeq m_{A}$ the allowed regions from $\tau \rightarrow \mu \nu \nu$ would be slightly larger. 
opposite sign for $\epsilon_{33}^{\ell}=0$ (neglecting flavor-violating couplings). However, the interference is constructive for $\epsilon_{33}^{\ell}>m_{\tau} / v$, allowing for an explanation with smaller values of $\tan \beta$ and/or heavier scalars. Note that for $\epsilon_{33}^{\ell}>m_{\tau} / v$ the $H$ contribution has the same sign as the SM contribution while the $A$ one has the opposite sign, so in our scenario it is a light $H$ that can solve the $\Delta a_{\mu}$ anomaly, as opposed to a light $A$ in the standard 2HDM- $X$ model. We show in Fig. 3 that $m_{H}$ must be smaller than $m_{A}$ for $\epsilon_{33}^{\ell}>m_{\tau} / v$. As seen above, $\epsilon_{33}^{\ell}>m_{\tau} / v$ is preferred by $\tau \rightarrow \mu \nu \nu$.

$h \rightarrow \mu \tau$ and $\tau \rightarrow \mu \gamma$ : So far, we have worked in the large $\tan \beta$ limit with $\alpha=0$. However, the decay $h \rightarrow \mu \tau$ can only appear for nonzero values of $\cos (\alpha-\beta)$. In this case additional Barr-Zee diagrams with gauge bosons or top quarks can contribute to $\tau \rightarrow \mu \gamma$ (see Ref. [86]). Therefore, the analysis is more involved than the one for the anomalous magnetic moment of the muon. However, we have checked in the case of $(g-2)_{\mu}$ (where the contributions are directly related to $\tau \rightarrow \mu \gamma)$ that $\cos (\alpha-\beta) \neq 0$ has actually only a small effect on the result.

To explain the CMS excess in $h \rightarrow \mu \tau$ [Eq. (7)], one needs a coupling strength of approximately

$$
\sin \alpha \tan \beta\left|\epsilon_{32}^{\ell}\right| \simeq 3.7 \times 10^{-3} .
$$

Nonzero values of $\epsilon_{32}^{\ell}$ then give rise to $\tau \rightarrow \mu \gamma$. The experimental upper limit for $\tau \rightarrow \mu \gamma$ is given by [106,107]

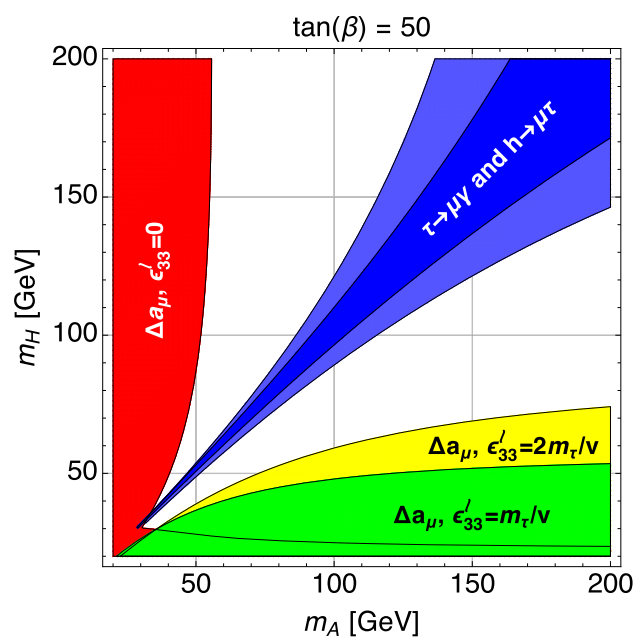

FIG. 3. The red, green, and yellow regions are the allowed regions in the $m_{A}-m_{H}$ plane from $(g-2)_{\mu}$ at the $2 \sigma$ level for $\tan \beta=50, m_{H^{+}}=200 \mathrm{GeV}, \cos (\alpha-\beta)=0.1$, and different values of $\epsilon_{33}^{\ell}$. The blue region is the allowed region (again at $2 \sigma)$ from $\tau \rightarrow \mu \gamma$ and $h \rightarrow \mu \tau$ for $\epsilon_{33}^{\ell}=2 m_{\tau} / v$ and $\cos (\alpha-\beta)=0.1$; light blue corresponds to $\cos (\alpha-\beta)=0.2$. The allowed region for $\Delta a_{\mu}$ covers the three possibilities $\epsilon_{32}^{\ell} \neq 0$, $\epsilon_{32}^{\ell}=\epsilon_{23}^{\ell} \neq 0$, and $\epsilon_{32}^{\ell}=-\epsilon_{23}^{\ell} \neq 0$, since the latter ones can give $m_{\tau} / m_{\mu}$ enhanced one-loop contributions. However, the effects turn out to be small, as $\epsilon_{32,32}^{\ell}$ is stringently constrained from $\tau \rightarrow \mu \gamma$. In addition, we checked that the effect of $\lambda_{H}$ is very small. The white regions are not compatible with experiment at the $2 \sigma$ level.

$$
\mathrm{BR}(\tau \rightarrow \mu \gamma) \leq 4.4 \times 10^{-8} \quad \text { at } 90 \% \text { C.L. }
$$

It is interesting to see if one can explain $h \rightarrow \mu \tau$ and $a_{\mu}$ simultaneously without violating bounds from $\tau \rightarrow \mu \gamma$. As the loop contributions to $\tau \rightarrow \mu \gamma$ are governed by the same Wilson coefficients as $a_{\mu}$, this turns out to be challenging. In Fig. 3 we show in blue and yellow the allowed regions in the $m_{A}-m_{H}$ plane for $\tau \rightarrow \mu \gamma, h \rightarrow \mu \tau$, and $a_{\mu}$ for $\epsilon_{33}^{\ell}=2 m_{\tau} / v$. As one can see, there is no overlap among all regions. There is an $m_{\tau} / m_{\mu}$ enhanced contribution to $(g-2)_{\mu}$ in the case $\epsilon_{23}^{\ell} \neq 0$ and $\epsilon_{32}^{\ell} \neq 0$. Even though we restricted ourselves in Eq. (10) to vanishing values of $\epsilon_{23}^{\ell}$, we checked that also for $\epsilon_{32}^{\ell}=\epsilon_{23}^{\ell}$ the effect in $a_{\mu}$ is small, taking into account the upper limit on $\epsilon_{32}^{\ell}=\epsilon_{23}^{\ell}$ from $\tau \rightarrow \mu \gamma$ while aiming at an explanation of $h \rightarrow \mu \tau$.

In principle, one might increase the coupling strength $\Gamma_{\mu \mu}^{H, A, H^{+}}$with the help of $\epsilon_{22}^{\ell}$. This would soften the tight relationship

$$
c_{R}^{\mu \tau} / c_{R}^{\mu \mu} \simeq \Gamma_{\mu \tau}^{H_{k}^{0}} / \Gamma_{\mu \mu}^{H_{k}^{0}} \simeq \epsilon_{32}^{\ell} v / m_{\mu}
$$

originating from the dominant Barr-Zee diagram with a $\tau$ loop, which causes the incompatibility of $a_{\mu}$ and $\tau \rightarrow \mu \gamma$. For the precise definitions of the Wilson coefficients $c_{R}^{\mu \tau}$, and $c_{R}^{\mu \mu}$ related to the Barr-Zee contributions we refer the reader to the Supplemental Material [86]. However, a large shift in $\Gamma_{\mu \mu}^{H, A, H^{+}}$from $\epsilon_{22}^{\ell} \ll m_{\mu} / v$ would mean fine tuning and also strongly affect $\tau \rightarrow \mu \nu \nu$. Therefore, we conclude that explaining $a_{\mu}$ and $h \rightarrow \mu \tau$ simultaneously is not impossible, but rather difficult and would involve fine tuning.

$t \rightarrow H c$ : For light values of $m_{H}$, as preferred by the anomalous magnetic moment of the muon, and nonzero values of $\epsilon_{32}^{u}$ as required by an explanation of $\mathcal{R}\left(D^{(*)}\right)$, the flavor changing top decay $t \rightarrow H c$ can have sizable branching ratios. In fact, as shown in Fig. 4 the branching ratio can easily be of the order of $10^{-2}$.

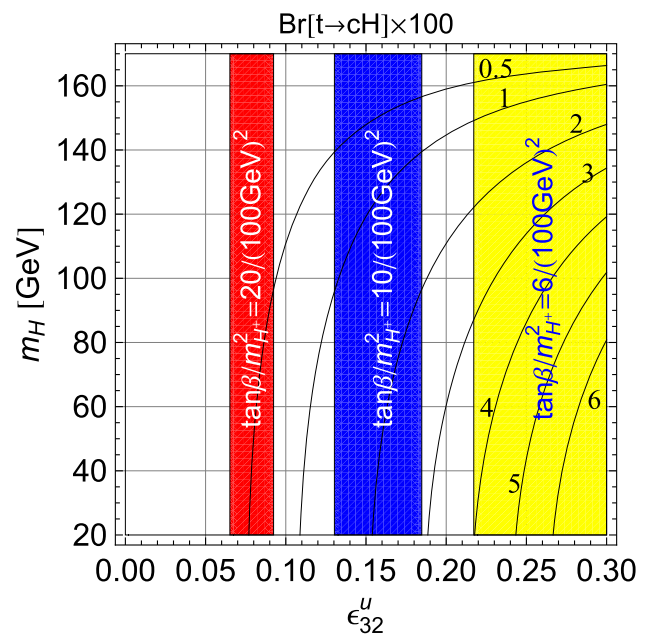

FIG. 4. The contour lines denote $\mathrm{BR}(t \rightarrow H c) \times 100$ as a function of $\epsilon_{32}^{u}$ and $m_{H}$. The colored regions are allowed by $\mathcal{R}(D)$ and $\mathcal{R}\left(D^{*}\right)$ for different values of $\tan \beta / m_{H^{+}}^{2}$. 
Conclusions. - In this Letter we addressed the measured anomalies in $\mathcal{R}\left(D^{(*)}\right)(3.8 \sigma), a_{\mu}(\sim 3 \sigma), \tau \rightarrow \mu \nu \nu(2.4 \sigma)$, and $h \rightarrow \mu \tau(2.4 \sigma)$ within a simple two-Higgs-doublet model. The Yukawa structure of our model is close to the lepton-specific 2HDM (type X), but with some additional Yukawa couplings involving third-generation fermions that give rise to the $b-c$ [necessary for $\mathcal{R}\left(D^{(*)}\right)$ ] and $\mu-\tau$ transitions (relevant for $h \rightarrow \mu \tau$ ) as well as corrections to $\tau \tau$ couplings (important for $\tau \rightarrow \mu \nu \nu$ ).

Let us summarize the logic of the Letter. (i) $\tau \rightarrow \mu \nu \nu$ prefers $\epsilon_{33}^{\ell} \geq m_{\tau} / v$. If one wants to account for the PDG average, also $\tan \beta>50$ is desirable. (ii) $a_{\mu}$ favors small values of $m_{H}$ for $\epsilon_{33}^{\ell} \geq m_{\tau} / v$ as in this case the Barr-Zee contribution with a $\tau$ loop has the correct sign and the diagrams involving a Higgs self-coupling can be relevant. (iii) $\mathcal{R}(D)$ and $\mathcal{R}\left(D^{*}\right)$ point towards quite large negative values of $\epsilon_{32}^{u}$ (of order -0.1). (iv) In the case of a light $H$ (as preferred by $a_{\mu}$ ), sizable decay rates for $t \rightarrow H c$ are possible if $\mathcal{R}(D)$ and $\mathcal{R}\left(D^{*}\right)$ are explained via $\epsilon_{32}^{u}$. This decay could be observed at the LHC in the process $p p \rightarrow \bar{t} t, t \rightarrow H c, H \rightarrow \tau \tau$. (v) $h \rightarrow \mu \tau$ can be explained using $\epsilon_{32}^{\ell}$. In this case $\alpha \neq 0$ and constraints from $\tau \rightarrow \mu \gamma$ arise. As the Barr-Zee contributions in $\tau \rightarrow \mu \gamma$ are directly correlated to the ones in $a_{\mu}$, a simultaneous explanation is difficult. (vi) If one attempts to explain $h \rightarrow \mu \tau$ (disregarding $a_{\mu}$ ), the exotic process $p p \rightarrow \bar{t} t, t \rightarrow H c, H \rightarrow \mu \tau$ can occur at the LHC.

Therefore, the future prospects are very promising: the decay $h \rightarrow \mu \tau$ implies rates for $\tau \rightarrow \mu \gamma$ in the reach of future experiments. More data on tau leptons are necessary to test our model, in particular $\tau \rightarrow \ell \nu \nu, \tau \rightarrow \mu \gamma$, and $h \rightarrow \tau \tau$. Furthermore, the light $H$ and the flavor-changing couplings required for $\mathcal{R}\left(D^{(*)}\right)$ lead to the decay $t \rightarrow H c$, followed by $H \rightarrow \tau \tau$ (or even $H \rightarrow \mu \tau$ ), which can be searched for at the LHC. While we did not attempt to find a symmetry realization of the pattern assumed for the $\epsilon_{i j}^{f}$ structures, it would be very interesting to find appropriate flavor symmetries to generate them dynamically, as the model works very well phenomenologically. An additional venue of interest would be the inclusion of dark matter in order to explain the Galactic center gamma-ray excess [108].

A. C. is supported by a Marie Curie Intra-European Fellowship of the European Community's 7th Framework Programme under Contract No. PIEF-GA-2012-326948. The work of J. H. is funded in part by IISN and by Belgian Science Policy (IAP VII/37). P. S. gratefully acknowledges financial support by the DFG (CRC 16, "Subnuclear Structure of Matter").

[1] P. Krawczyk and S. Pokorski, Phys. Rev. Lett. 60, 182 (1988).

[2] G. Bennett et al. (Muon $(g-2)$ Collaboration), Phys. Rev. D 73, 072003 (2006).
[3] K. Olive et al. (Particle Data Group), Chin. Phys. C 38, 090001 (2014).

[4] T. Aoyama, M. Hayakawa, T. Kinoshita, and M. Nio, Phys. Rev. Lett. 109, 111808 (2012).

[5] A. Czarnecki, B. Krause, and W. J. Marciano, Phys. Rev. D 52, R2619 (1995).

[6] A. Czarnecki, B. Krause, and W. J. Marciano, Phys. Rev. Lett. 76, 3267 (1996).

[7] C. Gnendiger, D. Stöckinger, and H. Stöckinger-Kim, Phys. Rev. D 88, 053005 (2013).

[8] M. Davier, A. Hoecker, B. Malaescu, and Z. Zhang, Eur. Phys. J. C 71, 1515 (2011).

[9] K. Hagiwara, R. Liao, A. D. Martin, D. Nomura, and T. Teubner, J. Phys. G 38, 085003 (2011).

[10] A. Kurz, T. Liu, P. Marquard, and M. Steinhauser, Phys. Lett. B 734, 144 (2014).

[11] F. Jegerlehner and A. Nyffeler, Phys. Rep. 477, 1 (2009).

[12] G. Colangelo, M. Hoferichter, A. Nyffeler, M. Passera, and P. Stoffer, Phys. Lett. B 735, 90 (2014).

[13] G. Colangelo, M. Hoferichter, M. Procura, and P. Stoffer, J. High Energy Phys. 09 (2014) 091.

[14] G. Colangelo, M. Hoferichter, B. Kubis, M. Procura, and P. Stoffer, Phys. Lett. B 738, 6 (2014).

[15] V. Pauk and M. Vanderhaeghen, Phys. Rev. D 90, 113012 (2014).

[16] G. Colangelo, M. Hoferichter, M. Procura, and P. Stoffer, J. High Energy Phys. 09 (2015) 074.

[17] M. Hayakawa, T. Blum, T. Izubuchi, and N. Yamada, Proc. Sci., LAT2005 (2006) 353.

[18] T. Blum, M. Hayakawa, and T. Izubuchi, Proc. Sci., LATTICE2012 (2012), 022.

[19] T. Blum, S. Chowdhury, M. Hayakawa, and T. Izubuchi, Phys. Rev. Lett. 114, 012001 (2015).

[20] J. Green, O. Gryniuk, G. von Hippel, H. B. Meyer, and V. Pascalutsa, Phys. Rev. Lett. 115, 222003 (2015).

[21] D. Stöckinger, J. Phys. G 34, R45 (2007).

[22] P. Langacker, Rev. Mod. Phys. 81, 1199 (2009).

[23] S. Baek, N. G. Deshpande, X. G. He, and P. Ko, Phys. Rev. D 64, 055006 (2001).

[24] E. Ma, D. P. Roy, and S. Roy, Phys. Lett. B 525, 101 (2002).

[25] S. N. Gninenko and N. V. Krasnikov, Phys. Lett. B 513, 119 (2001).

[26] M. Pospelov, Phys. Rev. D 80, 095002 (2009).

[27] J. Heeck and W. Rodejohann, Phys. Rev. D 84, 075007 (2011).

[28] K. Harigaya, T. Igari, M. M. Nojiri, M. Takeuchi, and K. Tobe, J. High Energy Phys. 03 (2014) 105.

[29] W. Altmannshofer, S. Gori, M. Pospelov, and I. Yavin, Phys. Rev. Lett. 113, 091801 (2014).

[30] D. Chakraverty, D. Choudhury, and A. Datta, Phys. Lett. B 506, 103 (2001).

[31] K. Cheung, Phys. Rev. D 64, 033001 (2001).

[32] A. Freitas, J. Lykken, S. Kell, and S. Westhoff, J. High Energy Phys. 05 (2014) 145 (2014); 09 (2014) 155(E).

[33] E. O. Iltan and H. Sundu, Acta Phys. Slovaca 53, 17 (2003).

[34] Y. Omura, E. Senaha, and K. Tobe, J. High Energy Phys. 05 (2015) 028. 
[35] A. Broggio, E. J. Chun, M. Passera, K. M. Patel, and S. K. Vempati, J. High Energy Phys. 11 (2014) 058.

[36] L. Wang and X.-F. Han, J. High Energy Phys. 05 (2015) 039.

[37] T. Abe, R. Sato, and K. Yagyu, J. High Energy Phys. 07 (2015) 064.

[38] J. Lees et al. (BABAR Collaboration), Phys. Rev. Lett. 109, 101802 (2012).

[39] T. Chur (Belle Collaboration), https://agenda.hepl.phys .nagoya-u.ac.jp/indico/getFile.py/access? contribId=22 \&sessionId $=4 \&$ resId $=0 \&$ materialId $=$ slides \& $\operatorname{confId}=170$ (2015).

[40] M. Huschle et al. (Belle Collaboration), Phys. Rev. D 92, 072014 (2015).

[41] R. Aaij et al. (LHCb Collaboration), Phys. Rev. Lett. 115, 111803 (2015).

[42] M. Rotondo, in Proceedings of Flavor Physics \& CP violation 2015 (FPCP 2015), Nagoya, Japan, May 2015, https://agenda.hepl.phys.nagoya-u.ac.jp/indico/getFile.py/ access ?contribId $=9 \&$ sessionId $=19 \&$ res $I d=0 \&$ materialId $=$ slides\&confId=170 (2015).

[43] J. F. Kamenik and F. Mescia, Phys. Rev. D 78, 014003 (2008).

[44] S. Fajfer, J. F. Kamenik, and I. Nisandzic, Phys. Rev. D 85, 094025 (2012).

[45] S. Fajfer, J. F. Kamenik, I. Nisandzic, and J. Zupan, Phys. Rev. Lett. 109, 161801 (2012).

[46] A. Crivellin, C. Greub, and A. Kokulu, Phys. Rev. D 86, 054014 (2012).

[47] A. Datta, M. Duraisamy, and D. Ghosh, Phys. Rev. D 86, 034027 (2012).

[48] A. Celis, M. Jung, X.-Q. Li, and A. Pich, J. High Energy Phys. 01 (2013) 054.

[49] A. Crivellin, C. Greub, and A. Kokulu, Phys. Rev. D 87, 094031 (2013).

[50] X.-Q. Li, Y.-D. Yang, and X.-B. Yuan, Phys. Rev. D 89, 054024 (2014).

[51] G. Faisel, Phys. Lett. B 731, 279 (2014).

[52] M. Atoui, V. Morénas, D. Bečirevic, and F. Sanfilippo, Eur. Phys. J. C 74, 2861 (2014).

[53] Y. Sakaki, R. Watanabe, M. Tanaka, and A. Tayduganov, Phys. Rev. D 88, 094012 (2013).

[54] I. Doršner, S. Fajfer, N. Košnik, and I. Nišandžić, J. High Energy Phys. 11 (2013) 084.

[55] P. Biancofiore, P. Colangelo, and F. De Fazio, Phys. Rev. D 89, 095018 (2014).

[56] R. Alonso, B. Grinstein, and J. M. Camalich, J. High Energy Phys. 10 (2015) 184.

[57] A. Greljo, G. Isidori, and D. Marzocca, J. High Energy Phys. 07 (2015) 142.

[58] L. Calibbi, A. Crivellin, and T. Ota, Phys. Rev. Lett. 115, 181801 (2015).

[59] M. Freytsis, Z. Ligeti, and J. T. Ruderman, Phys. Rev. D 92, 054018 (2015).

[60] M. Tanaka and R. Watanabe, Phys. Rev. D 87, 034028 (2013).

[61] M. Krawczyk and D. Temes, Eur. Phys. J. C 44, 435 (2005).

[62] Y. Amhis et al. (Heavy Flavor Averaging Group (HFAG)), arXiv:1412.7515.
[63] W. Hollik and T. Sack, Phys. Lett. B 284, 427 (1992).

[64] M. Aoki, S. Kanemura, K. Tsumura, and K. Yagyu, Phys. Rev. D 80, 015017 (2009).

[65] A. Crivellin, L. Hofer, J. Matias, U. Nierste, S. Pokorski, and J. Rosiek, Phys. Rev. D 92, 054013 (2015).

[66] V. Khachatryan et al. (CMS Collaboration), Phys. Lett. B749, 337 (2015).

[67] M. D. Campos, A. E. Carcamo Hernández, H. Päs, and E. Schumacher, Phys. Rev. D 91, 116011 (2015).

[68] D. Aristizabal Sierra and A. Vicente, Phys. Rev. D 90, 115004 (2014).

[69] J. Heeck, M. Holthausen, W. Rodejohann, and Y. Shimizu, Nucl. Phys. B896, 281 (2015).

[70] A. Crivellin, G. D’Ambrosio, and J. Heeck, Phys. Rev. Lett. 114, 151801 (2015).

[71] I. Dorsner, S. Fajfer, A. Greljo, J. F. Kamenik, N. Kosnik, and I. Nišandžić, J. High Energy Phys. 06 (2015) 108.

[72] A. Crivellin, G. D'Ambrosio, and J. Heeck, Phys. Rev. D 91, 075006 (2015).

[73] C.-W. Chiang, H. Fukuda, M. Takeuchi, and T. T. Yanagida, J. High Energy Phys. 11 (2015) 057.

[74] T. Miki et al., arXiv:hep-ph/0210051.

[75] El Kaffas, P. Osland, and O. M. Ogreid, Phys. Rev. D 76, 095001 (2007).

[76] O. Deschamps, S. Monteil, V. Niess, S. Descotes-Genon, S. TJampens, and V. Tisserand, Phys. Rev. D 82, 073012 (2010).

[77] G. Branco, P. Ferreira, L. Lavoura, M. Rebelo, M. Sher, and J. P. Silva, Phys. Rep. 516, 1 (2012).

[78] G. D’Ambrosio, G. F. Giudice, G. Isidori, and A. Strumia, Nucl. Phys. B645, 155 (2002).

[79] A. J. Buras, M. V. Carlucci, S. Gori, and G. Isidori, J. High Energy Phys. 10 (2010) 009.

[80] G. Blankenburg and G. Isidori, Eur. Phys. J. Plus 127, 85 (2012).

[81] A. Pich and P. Tuzon, Phys. Rev. D 80, 091702 (2009).

[82] M. Jung, A. Pich, and P. Tuzon, J. High Energy Phys. 11 (2010) 003.

[83] S. L. Glashow and S. Weinberg, Phys. Rev. D 15, 1958 (1977).

[84] M. Misiak, H. Asatrian, R. Boughezal, M. Czakon, T. Ewerth et al., Phys. Rev. Lett. 114, 221801 (2015).

[85] V. Khachatryan et al. (CMS Collaboration), J. High Energy Phys. 10 (2014) 160.

[86] See Supplemental Material athttp://link.aps.org/ supplemental/10.1103/PhysRevLett.116.081801, which includes Refs. [87-104], for the formulas for the various processes and the related Wilson coefficients.

[87] W.-S. Hou, Phys. Rev. D 48, 2342 (1993).

[88] A. Crivellin and S. Pokorski, Phys. Rev. Lett. 114, 011802 (2015).

[89] A. Akeroyd and S. Recksiegel, J. Phys. G 29, 2311 (2003).

[90] Y. Sakaki and H. Tanaka, Phys. Rev. D 87, 054002 (2013).

[91] S. M. Barr and A. Zee, Phys. Rev. Lett. 65, 21 (1990); 65, 2920(E) (1990).

[92] D. Chang, W. S. Hou, and W.-Y. Keung, Phys. Rev. D 48, 217 (1993).

[93] J. Hisano, M. Nagai, and P. Paradisi, Phys. Lett. B 642, 510 (2006).

[94] M. Jung and A. Pich, J. High Energy Phys. 04 (2014) 076. 
[95] T. Abe, J. Hisano, T. Kitahara, and K. Tobioka, J. High Energy Phys. 01 (2014) 106.

[96] V. Ilisie, J. High Energy Phys. 04 (2015) 077.

[97] S. Chatrchyan et al. (CMS Collaboration), J. High Energy Phys. 05 (2014) 104.

[98] G. Aad et al. (ATLAS), J. High Energy Phys. 04 (2015) 117.

[99] J. F. Gunion, H. E. Haber, G. L. Kane, and S. Dawson, Front. Phys. 80, 1 (2000).

[100] J. Cao, P. Wan, L. Wu, and J. M. Yang, Phys. Rev. D 80, 071701 (2009).

[101] T. Goto, R. Kitano, and S. Mori, Phys. Rev. D 92, 075021 (2015).
[102] S. Davidson, S. Lacroix, and P. Verdier, J. High Energy Phys. 09 (2012) 092.

[103] D. Chang, W.-F. Chang, C.-H. Chou, and W.-Y. Keung, Phys. Rev. D 63, 091301 (2001).

[104] W. Skiba and J. Kalinowski, Nucl. Phys. B404, 3 (1993).

[105] A. Crivellin and L. Mercolli, Phys. Rev. D 84, 114005 (2011).

[106] B. Aubert et al. (BABAR Collaboration), Phys. Rev. Lett. 104, 021802 (2010).

[107] K. Hayasaka et al. (Belle), Phys. Lett. B 666, 16 (2008).

[108] A. Hektor, K. Kannike, and L. Marzola, J. Cosmol. Astropart. Phys. 10 (2015) 025. 